\title{
Bringing VISION to Nuclear Medicine: Accelerating Evidence and Changing Paradigms with Theranostics
}

\author{
Michael S. Hofman \\ Molecular Imaging and Therapeutic Nuclear Medicine, Peter MacCallum Cancer Centre, and Sir Peter MacCallum Department of \\ Oncology, University of Melbourne, Melbourne, Australia
}

I (PSMA) as a game-changing target for nuclear medicine at the 2011 European Association of Nuclear Medicine meeting in Birmingham. The target had been identified 2 decades beforehand (1,2). A new approach using radiolabeled small-molecule ligands that target the extracellular domain of the PSMA receptor, however, showed striking tumor-to-background contrast in both preclinical models with ${ }^{99 \mathrm{~m}} \mathrm{Tc}$ or ${ }^{124} \mathrm{I} /{ }^{131} \mathrm{I}$ and in a ${ }^{68} \mathrm{Ga}$ first-in-humans image of a patient with prostate cancer. I recorded PSMA as my "Image of the Conference." The images provided the clarity needed in order to be rapidly adopted by groups into clinical practice and trials in both imaging and therapy. A decade has passed, and we now have 2 Food and Drug Administration-approved PSMA ligands, ${ }^{68} \mathrm{Ga}$-PSMA-11 and ${ }^{18}$ F-DCFPyL. And we also have VISION, a phase 3 trial of radioligand therapy, that has led to Food and Drug Administration breakthrough approval of ${ }^{177}$ Lu-PSMA-617.

The VISION trial, presented at the American Society of Clinical Oncology 2021 meeting and recently published in The New England Journal of Medicine (3), included men with metastatic castration-resistant prostate cancer who had previous treatment with at least 1 taxane chemotherapy and 1 androgen receptor pathway inhibitor. Thirteen percent were excluded after ${ }^{68} \mathrm{Ga}-\mathrm{P}-$ SMA-11 PET/CT selection; 551 men were randomized to ${ }^{177} \mathrm{Lu}-$ PSMA-617 and 280 to the standard of care. Although the standard of care can be criticized for being protocol-defined and potentially limiting optimal clinical care options, the trial was designed with a goal of Food and Drug Administration approval and the results will enable widespread, global availability for men with prostate cancer. This availability is expected to follow, given the improvement in overall survival of 15.3 mo with ${ }^{177}$ Lu-PSMA-617, compared with 11.3 mo with the standard of care. This improvement was consistent across prespecified stratification factors, including lactate dehydrogenase, liver metastases, and androgen-receptor pathway inhibitors planned as part of the standard of care. Other endpoints, such as response on CT (RECIST response, $42 \%$ vs. 3\%) and a prostatespecific antigen decline of over $50 \%$ ( $46 \%$ vs. $7 \%)$, also impressively favor ${ }^{177}$ Lu-PSMA-617.

Like many other types of radionuclide therapy that are now widely available, ${ }^{177} \mathrm{Lu}-\mathrm{PSMA}-617$ has followed a development

Received Jul. 11, 2021; revision accepted Jul. 16, 2021.

For correspondence or reprints, contact Michael S. Hofman (michael. hofman@petermac.org).

Published online August 12, 2021.

COPYRIGHT (C) 2021 by the Society of Nuclear Medicine and Molecular Imaging. DOI: 10.2967/jnumed.121.262890 pathway that is unusual compared with conventional pharmaceuticals. The very first treatments were compassionate-access treatments at Bad Berka (4) and the University of Heidelberg (5) in Germany in men who had progressed after standard therapies. The unique ability to see what you treat with theranostics enabled the confidence to administer a treatment never tested before in a human. Years of experience with ${ }^{68} \mathrm{Ga} /{ }^{177} \mathrm{Lu}$-DOTATATE in neuroendocrine tumors enabled estimation of an appropriate administered radioactivity. Posttreatment dosimetry instantly validated high tumor targeting with low normal-organ uptake (6). Such a direct mechanistic treatment paradigm has features more analogous to externalbeam radiotherapy, enabling rapid translation to the clinic.

At the Peter MacCallum Cancer Centre, we designed a prospective phase II study in 2014. Our vision was that a small prospective trial would provide the type of evidence required to evaluate activity and safety and to move this treatment into the mainstream. The results of this 30-patient trial were published in Lancet Oncology $(7,8)$ and are currently the topic of the top 10 most highly cited articles in the journal since 2008. Without a commercial sponsor but with support from the ANZUP Clinical Trials Group and grant funding, these results led directly to the first randomized controlled trial of ${ }^{177} \mathrm{Lu}-\mathrm{PSMA}-617$, the TheraP trial. ABX, manufacturer and owner of the PSMA-617 intellectual property at the time, and ANSTO, which manufactures ${ }^{177} \mathrm{Lu}$ in Australia, kindly agreed to the support trial. Our expanded 50-patient cohort (7) provided Endocyte with compelling phase 2 evidence contributing to its purchase of PSMA-617 and design of the phase 3 VISION trial (9). Around a year later, Novartis purchased Endocyte and spearheaded global commercialization.

We now have 2 randomized trials of ${ }^{177} \mathrm{Lu}-\mathrm{PSMA}-617$ providing complementary evidence. VISION provides definitive survival data on men who have exhausted current therapeutic options. The TheraP trial places PSMA theranostics 1 step earlier by comparing with cabazitaxel (10), showing greater efficacy, lower toxicity, and better patient-reported outcomes. VISION demonstrates efficacy in a broader population, whereas TheraP used quantitative PET and also ${ }^{18} \mathrm{~F}$-FDG PET/CT for patient selection. It remains an open question whether men with relatively low-intensity PSMA uptake or ${ }^{18} \mathrm{~F}$ FDG-positive PSMA-negative disease benefit from this treatment.

We recently celebrated the anniversary of the first administration of radioiodine by Saul Hertz on March 31, 1941. Eighty years later, the nuclear medicine community continues to innovate with truly personalized medicine. As a last line of treatment, outcomes with ${ }^{177} \mathrm{Lu}$-PSMA remain modest for many men, although some experience truly exceptional and durable responses. I am hopeful 
that multiple efforts are also under way to evaluate novel combinations and that use of ${ }^{177}$ Lu-PSMA earlier, even as a first line of treatment $(11,12)$, will further improve outcomes for men with prostate cancer.

\section{DISCLOSURE}

Michael Hofman is supported by the Prostate Cancer Foundation (PCF), funding the Prostate Cancer Theranostics and Imaging Centre of Excellence (ProTIC), with additional grant funding from Movember, Victorian Cancer Agency (VCA), and the U.S. Department of Defense. Michael Hofman has also received speaker fees, travel support, or fees for advisory board participation from Astella, AstraZeneca, Janssen, Merck/MSD, Mundipharma, and Point Biopharma, as well as research support from Endocyte and Advanced Accelerator Applications (Novartis companies). Michael Hofman was also the principal investigator of the TheraP study, funded by the Prostate Cancer Foundation of Australia (PCFA), with community support from Movember, It's a Bloke Thing Foundation, and Can4Cancer. ANSTO supplied no-carrier-added ${ }^{177} \mathrm{Lu}$. No other potential conflict of interest relevant to this article was reported.

\section{REFERENCES}

1. Liu H, Moy P, Kim S, et al. Monoclonal antibodies to the extracellular domain of prostate-specific membrane antigen also react with tumor vascular endothelium. Cancer Res. 1997;57:3629-3634.
2. Israeli RS, Powell CT, Fair WR, Heston WD. Molecular cloning of a complementary DNA encoding a prostate-specific membrane antigen. Cancer Res. 1993;53:227-230.

3. Sartor O, de Bono J, Chi KN, et al. Lutetium-177-PSMA-617 for metastatic castration-resistant prostate cancer. $N$ Engl J Med. 2021;385:1091-1103.

4. Weineisen M, Schottelius M, Simecek J, et al. ${ }^{68} \mathrm{Ga}$ - and ${ }^{177}$ Lu-labeled PSMA I\&T: optimization of a PSMA-targeted theranostic concept and first proof-of-concept human studies. J Nucl Med. 2015;56:1169-1176.

5. Afshar-Oromieh A, Hetzheim H, Kratochwil C, et al. The theranostic PSMA ligand PSMA-617 in the diagnosis of prostate cancer by PET/CT: biodistribution in humans, radiation dosimetry, and first evaluation of tumor lesions. $J$ Nucl Med. 2015;56:1697-1705.

6. Violet J, Jackson P, Ferdinandus J, et al. Dosimetry of Lu-PSMA-617 in metastatic castration-resistant prostate cancer: correlations between pretherapeutic imaging and whole-body tumor dosimetry with treatment outcomes. J Nucl Med. 2019;60:517-523.

7. Violet J, Sandhu S, Iravani A, et al. Long-term follow-up and outcomes of retreatment in an expanded 50-patient single-center phase II prospective trial of Lu-PSMA-617 theranostics in metastatic castration-resistant prostate cancer. J Nucl Med. 2020;61:857-865.

8. Hofman MS, Violet J, Hicks RJ, et al. [177Lu]-PSMA-617 radionuclide treatment in patients with metastatic castration-resistant prostate cancer (LuPSMA trial): a single-centre, single-arm, phase 2 study. Lancet Oncol. 2018;19:825-833.

9. Sherman M, Levine R. Nuclear medicine and Wall Street: an evolving relationship. J Nucl Med. 2019;60(suppl):20S-24S.

10. de Wit R, de Bono J, Sternberg CN, et al. Cabazitaxel versus abiraterone or enzalutamide in metastatic prostate cancer. N Engl J Med. 2019;381:2506-2518.

11. Dhiantravan N, Violet J, Eapen R, et al. Clinical trial protocol for LuTectomy: a single-arm study of the dosimetry, safety, and potential benefit of Lu-PSMA-617 prior to prostatectomy. Eur Urol Focus. 2021;7:234-237.

12. Dhiantravan N, Emmett L, Joshua AM, et al. UpFrontPSMA: a randomized phase 2 study of sequential Lu-PSMA-617 and docetaxel vs docetaxel in metastatic hormone-naïve prostate cancer (clinical trial protocol). BJU Int. 2021; 128:331-342. 